LRH: JAGM DE VISSER, J HERMISSON ET AL.

RRH: EVOLUTION OF ROBUSTNESS

\title{
PERSPECTIVE: EVOLUTION AND DETECTION OF GENETIC ROBUSTNESS
}

J. Arjan G.M. de Visser ${ }^{1,}$ *, Joachim Hermisson ${ }^{2,}{ }^{*}, \$$, Günter P. Wagner ${ }^{3, \$}$, Lauren Ancel Meyers ${ }^{4}$, Homayoun Bagheri-Chaichian ${ }^{5}$, Jeffrey L. Blanchard ${ }^{6}$, Lin Chao ${ }^{7}$, James M. Cheverud ${ }^{8}$, Santiago F. Elena ${ }^{9}$, Walter Fontana ${ }^{5}$, Greg Gibson ${ }^{10}$, Thomas F. Hansen ${ }^{11}$, David Krakauer ${ }^{5}$, Richard C. Lewontin ${ }^{12}$, Charles Ofria ${ }^{13}$, Sean H. Rice $^{3}$, George von Dassow ${ }^{14}$, Andreas Wagner ${ }^{15}$, and Michael C. Whitlock ${ }^{16}$

* Corresponding authors

$\$$ Organizers of the workshop Detection and Evolution of Genetic Robustness, April 23-25, 2002 at the Santa Fe Institute, Santa Fe, NM

${ }^{1}$ Department of Genetics, Wageningen University, Wageningen, The Netherlands, Email:arjan.devisser@wur.nl

${ }^{2}$ Department Biology II, Ludwig-Maximilians-University Munich, Germany, E-mail: hermisson@zi.biologie.uni-muenchen.de

${ }^{3}$ Department of Ecology and Evolutionary Biology, Yale University, New Haven, CT

${ }^{4}$ Integrative Biology, The University of Texas at Austin, Austin, TX

${ }^{5}$ Santa Fe Institute, Santa Fe, NM

${ }^{6}$ National Center for Genome Resources, Santa Fe, NM

${ }^{7}$ University of California/San Diego, La Jolla, CA

${ }^{8}$ Department of Anatomy \& Neurobiology, Washington University, St. Louis, MO

${ }^{9}$ Instituto de Biologia Molecular y Celular de Plantas, CSIC, Valencia, Spain

${ }^{10}$ Department of Genetics, North Carolina State University, Raleigh, NC

${ }^{11}$ Department of Biological Sciences, Florida State University, Tallahassee, FL

${ }^{12}$ Museum of Comparative Zoology \& Department of Population Biology, Harvard

University, Cambridge, MA

${ }^{13}$ Center for Microbial Ecology, Michigan State University, East Lansing, MI

${ }^{14}$ Friday Harbor Labs, University of Washington, Friday Harbor, WA

${ }^{15}$ Department of Biology, University of New Mexico, Albuquerque, NM

${ }^{16}$ Department of Zoology, University of British Columbia, Vancouver, Canada 
Abstract. - Robustness is the invariance of phenotypes in the face of perturbation. The robustness of phenotypes appears at various levels of biological organization, including gene expression, protein folding, metabolic flux, physiological homeostasis, development, and even organismal fitness. The mechanisms underlying robustness are diverse, ranging from thermodynamic stability at the RNA and protein level to behavior at the organismal level. Phenotypes can be robust either against heritable perturbations (e.g. mutations) or non-heritable perturbations (e.g. the weather). Here we primarily focus on the first kind of robustness - genetic robustness - and survey three growing avenues of research: (1) measuring genetic robustness in nature and in the laboratory, (2) understanding the evolution of genetic robustness, and (3) exploring the implications of genetic robustness for future evolution.

Key words. - Genetic robustness, environmental robustness, canalization, epistasis, evolvability

One of the most intriguing revelations of modern genetics is the ubiquity of epistatic effects. That is, genes may have an effect on the phenotype, but this effect strongly depends on other genes in the genome. Hence genetic effects can be larger in one genetic background and smaller in another. Wild type genotypes often harbor large amounts of 'hidden' genetic variation (or 'potential' variation, cf. Gibson et al. 1999) that is only expressed when the genetic background changes. In yeast, for example, a large fraction of null mutations (up to 50\%) hardly affects fitness because the function of the knocked-out gene is compensated by other genes under the growth conditions applied (Thatcher et al. 1997). These observations of an amazing resilience of phenotypes with respect to genetic variation provide the ground for the idea of genetic robustness. This phenomenon is really one facet of a larger problem determining the genetic architecture of so-called complex phenotypic traits that vary through strong interactions among the contributing genes (e.g. Wade 2002).

But what is the origin of this observed robustness? Is it merely an "accident", or a consequence of natural selection in the face of mutations and environmental variation? And what are the evolutionary consequences of robustness? The amount of phenotypic diversity within or among populations can vary significantly. For example, scutellar bristle number in Drosophila melanogaster rarely deviates from the canonical four-bristles pattern, yet bristle number varies between species. Other characters, such as floral symmetry, may be highly conserved among species of a particular clade. While some of the variation may be due to differences in the selective forces acting on the character, the ubiquity of gene interaction and the potential for robust genotypes raises the intriguing possibility that other factors may play a role as well. Could prior selection have selected for genotypes that make a trait genetically robust and thus decrease its evolvability? Might it be possible to 'engineer' a genotype that makes a desirable phenotypic trait robust, for example, corn yield or the production of a growth hormone in milk? In both basic evolutionary theory and biotechnology, a proper understanding of the mechanisms and population genetic principles of genetic robustness is essential.

Following the classical work of Waddington and Schmalhausen and their contemporaries, research on robustness (called canalization by Waddington) experienced a decline during the 1970's and 1980's. This may have simply been a consequence of technical limitations in the pre-molecular era of genetics, a situation 
that has dramatically changed since the early 1990's. As powerful molecular techniques to track and manipulate genotypes become routine, a renewed interest in the issue of genetic robustness of phenotypic traits has surfaced. Even branches of biology that traditionally avoided evolutionary speculation have turned their attention to genetic robustness. For example, the recent discoveries of functional robustness in cellular mechanisms has prompted Gerhart and Kirschner to make robustness and its evolution the central topic of their book "Cells, Embryos and Evolution" (1997). Hartwell, primarily known for his work on cell cycle regulation, has summarized the surprisingly mild effects of many knockout genotypes and speculated as to the causes and the mechanisms of such genetic robustness (Hartman et al. 2001). Lindquist, Rutherford and collaborators, working on molecular chaperons, have addressed the context dependency of genetic effects, and conjectured the existence of 'capacitors' of genetic change (e.g. Rutherford and Lindquist 1998). At the same time, recent computational modeling of genetic regulatory and macro-molecules suggests a rich potential for evolving phenotypic traits with robustness against genetic and environmental perturbations. Finally, novel mathematical approaches in population genetics have also illuminated the population genetic mechanisms that may lead to genetic robustness by natural selection (for references see Gibson and Wagner 2000). These developments have occurred rapidly and with very little exchange among the different disciplines. This motivated a workshop at the Santa Fe Institute, supported by the Packard Foundation Program on Robustness, which brought together experimentalists and theorists to discuss these discoveries and the future of robustness research.

This perspective summarizes the theories about the evolution of genetic robustness and the new ideas that emerged during the workshop (and which thus belong to all participants of the workshop as well as the wider scientific community). In what follows, we address three fundamental questions: Under what circumstances and for which traits do we expect robustness to evolve? What are the evolutionary consequences of robustness? How can robustness be detected and measured?

\section{GENETIC AND ENVIRONMENTAL ROBUSTNESS}

Phenotypic robustness is the reduced sensitivity of a phenotype (defined here as any measurable aspect of an organism resulting from the expression of its genes) with respect to perturbations in the parameters (genetic and environmental) that affect its expression. It is important to distinguish between the buffering mechanism and the source of the perturbations. There are many buffering mechanisms, ranging from molecular to behavioral. Here, we consider only mechanisms that are heritable, and that may therefore evolve. On the other hand, the perturbations against which the phenotype is buffered may or may not be passed on to the progeny. This leads to the important distinction between environmental and genetic robustness.

\section{Environmental Robustness}

Taken in a broad sense, environmental robustness refers to any kind of buffering against non-heritable perturbations. These may be external environmental factors like temperature or salinity or internal factors like developmental noise caused 
by fluctuation in the concentration of relevant gene products. (Environmental robustness, as defined here, thus includes environmental canalization and developmental stability in the terminology of Hallgrímsson et al. 2002). One measure of environmental robustness is the inverse of the environmental variance, $V_{\mathrm{e}}$, which results from external environmental perturbations (see EXPERIMENTAL DETECTION OF ROBUSTNESS).

There is no reason to believe that a single buffering mechanism acts indiscriminately against all sources of perturbation. In fact, there are many mechanisms by which the stability of some trait or function is maintained in the face of environmental perturbations (cf. Ancel Meyers and Bull 2002). Many of these mechanisms are behavioral adaptations, such as habitat selection or nest building, which reduce the exposure to environmental fluctuations. Yet, these behavioral patterns are unlikely to buffer against the effects of developmental noise. Similarly, molecular buffering mechanisms have been found to confer robustness only against a subset of environmental sources of perturbation. Studying the sternopleural bristle number and the wing-vein length in Drosophila melanogaster, Rutherford (2000) found that heat-shock protein Hsp90, which provides robustness against perturbations in the external environment, does not suppress developmental noise. More precisely, Hsp90 does not affect the fluctuating asymmetry (FA) - the nondirectional variation in subtle differences between left and right sides of bilateral characters. Conversely, Scharloo (1988) was able to increase developmental stability (measured as inverse FA) in scutellar bristle number in a high-bristle line of $D$. melanogaster, without affecting the environmental stability (among-fly environmental variance) of the same trait. A significant positive correlation of FA and $V_{\mathrm{e}}$ was found for $262 \mathrm{limb}$ traits in fetal mice, suggesting an "extensive overlap" of buffering mechanisms for development and external environment (Hallgrímsson et al. 2002). However, this correlation could also be due to differences in the strength of selection on these traits (leading to enhanced buffering for traits under stronger selection). Moreover, variation in the number of genes encoding the various traits could cause a similar correlation (cf. section Comparative Approach: Genetic Robustness, paragraph Lack of reference and control).

Since environmental robustness confers stability to a phenotype in a variable environment, it will be favored by natural selection only when the optimum phenotype remains the same under these environmental variations. When the optimum phenotype depends on the environment, we expect selection for phenotypic plasticity, where the trait mean tracks the optimum. Often, however, phenotypic plasticity of a trait will entail environmental robustness at a higher phenotypic level (such as viability or fitness, see below). This underscores that environmental robustness and phenotypic plasticity are really two cases of the same phenomenon: evolution of the dependency of phenotype on some environmental factor. From an evolutionary point of view, both are organismal traits just like others, and are most easily explained as adaptations to an ever-changing world.

\section{Genetic Robustness}

This perspective primarily addresses genetic robustness, which, in the broad sense, refers to the constancy of the phenotype in the face of heritable perturbations (genetic or epigenetic). To be more specific, we focus on recurrent mutations 
('mutational robustness'). In this case, a reasonable measure of genetic robustness of a trait is the inverse of the mutational variance, $V_{\mathrm{m}}$, of this trait, i.e. the phenotypic variation caused by the input of new mutations. Assuming no variation in the molecular mutation rate, a state is called robust relative to an (ancestral) reference state if it has reduced $V_{\mathrm{m}}$. Many mechanisms of genetic robustness act in developmental or metabolic pathways, where they buffer the phenotype against the expression of mutations. As in the case of environmental robustness, however, mechanisms may also be behavioral (e.g. animals choosing their surroundings according to genetically determined body color). They may or may not act against non-heritable perturbations at the same time. If they do, this can have important consequences for the evolution of robustness (see Congruent below). Since phenotypically expressed genetic variation is the fuel of evolution, buffering mechanisms will necessarily influence its further course. In this sense, genetic robustness is similar to a 'genetic system', like recombination and mutation rates, which affect the fraction of all genetic variation that is useful for adaptation. Genetic robustness is thus critical to our understanding of evolution.

\section{TYPES OF GENETIC ROBUSTNESS}

While the short-term consequence - the maintenance of an optimal phenotype in the face of mutations - is clear and of considerable evolutionary importance, its evolutionary origins are far less clear. The theories addressing this problem can be grouped into three classes: adaptive, intrinsic, and congruent. As it turns out, these categories reflect the positions of the founding fathers of modern population genetics, R.A. Fisher, J.B.S. Haldane, and S. Wright, in their famous controversy over the evolution of dominance (cf. Mayo and Bürger 1997).

Dominance can be understood as a special case of genetic robustness at the level of a single gene, where a dominant phenotype is more robust against mutational (and perhaps environmental) perturbations than a recessive phenotype. It is thus not surprising that the evolutionary theories for dominance parallel those for genetic robustness in general. As we will see below, however, the dominance hypotheses must be revised for the general phenomenon of phenotypic robustness.

\section{Adaptive}

In a Darwinian tradition, the most straightforward explanation for the evolution of robustness is adaptationist. Robustness might simply evolve because it increases the fitness of the genotype involved. The idea is as intuitive as it is simple: for a well-adapted trait, almost all mutations lead to deviations from the optimum. Any mechanism that buffers the trait by decreasing the phenotypic effect of mutations should therefore be favored by natural selection. In the debate on dominance, R.A. Fisher took this position (1928), claiming that dominance evolves by the direct selection on modifiers. For phenotypic (genetic and environmental) robustness, the adaptive hypothesis goes back to the original work of Schmalhausen (1949), Waddington (1957), and Rendel (1959). Canalization as envisioned by Waddington evolves due to its own selective advantage and can thus be defined as adaptive robustness. Note, however, that the definition of canalization varies considerably in 
the literature (cf. Debat and David 2001 for an overview). Sometimes the term is used only for developmental buffering (e.g. Hallgrímsson et al. 2002). In order to emphasize a broader scope that also includes buffering by other mechanisms (such as behavior), we will use the term adaptive robustness in the following.

\section{Intrinsic}

Not every phenotype that evolves is, taken by itself, the product of selective optimization. Due to variational constraints, phenotypic properties often result as non-adaptive correlated side effects of the evolution of some other property. In particular, genetic robustness may evolve simply because the buffering of a character with respect to mutations is the necessary or likely consequence of character adaptation itself. In the context of dominance, $S$. Wright held this view $(1929,1934)$, claiming that dominance results as a passive consequence of enzyme biochemistry and the selection for increased metabolic flux (the primary character). Due to saturation relationships between enzyme activity and the flux through a metabolic pathway, the flux will typically approach its maximum asymptotically, where variation in enzyme activity has little effect. Nonetheless, Wright thought that dominance could be modified and subject to evolution (Wright 1977). Later, Kacser and Burns (1981) took a uniquely physiological position. They argued that due to the constraints of multi-enzyme biochemistry, dominance is an inevitable property of metabolic pathways and does not require an evolutionary explanation. Consequently, the view that dominance is an inevitable property of metabolic pathways has been accepted by several scientists (e.g. Orr 1991; Keightley 1996; Porteus 1996; Meiklejohn and Hartl 2002). Arguments to the contrary, however, have also been made (Cornish-Bowden 1987; Savageau 1992; Bourguet 1999; Omholt et al. 2000; Bagheri-Chaichian 2001). For genetic robustness in general, one can similarly argue that robustness is intrinsic to the optimization of some phenotypes, for example when several loci contribute to the steady-state metabolic flux, or when the activity of an enzyme is controlled by feed-back from its end-product (see PROXIMATE CAUSES OF ROBUSTNESS).

\section{Congruent}

A third view posits a correlation between the mechanisms of genetic and environmental robustness. Genetic robustness may thus evolve as a correlated side effect of the evolution for environmental robustness. Since environmental perturbations often have a higher frequency and impact on fitness, they serve as the driving force. As in the adaptive case, character robustness can be separated from, and is secondary to, character adaptation itself, but, as in the intrinsic case, genetic robustness does not evolve for its own sake. In the dominance controversy, this mirrors the idea of J.B.S. Haldane (1930), who argued that dominance results from safety-factors (see also Muller 1932, and later Wright 1977). According to Haldane, the wild type allele evolves to produce elevated amounts of enzyme in order to ensure optimal function of wild type homozygotes under unusual environmental conditions. As a consequence, the levels of enzyme concentration that can be produced by heterozygote mutants are often sufficient under normal conditions. This is then perceived as dominance of the wild type allele. For robustness mechanisms in 
general, correlated evolution of genetic and environmental robustness has been proposed in Wagner et al. (1997). A correlation between the phenotypic effects of genetic and environmental perturbations was called plastogenetic congruence by Ancel and Fontana (2000), who observed the concerted evolution of environmental and genetic robustness in theoretical work on RNA folding.

\section{SCENARIOS FOR THE EVOLUTION OF GENETIC ROBUSTNESS}

We now discuss in more detail several scenarios in which we would expect the adaptive, intrinsic, or congruent evolution of genetic robustness.

\section{Adaptive Scenarios}

Under which evolutionary conditions will genetic robustness have a substantial selective advantage? The selective advantage of a buffering gene is given by the sum of the negative fitness effects of the mutations it buffers against, minus any costs associated with the gene. Here we discuss several key evolutionary parameters that affect this calculation.

\section{Mutation rate}

A high mutation rate is perhaps the most important prerequisite for adaptive genetic robustness. In most situations, the selective advantage of mechanisms that buffer against mutations is simply proportional to the mutation rate $U$ of the character (or the buffered unit) under consideration. As we will discuss below (Strength of selection), the fitness effects of the mutations have much less effect. This holds true for clonally reproducing organisms, where all the genetic perturbations must be created by new mutations. In sexual (and recombining) populations, the evolution of robustness may also be driven by the standing genetic variation rather than solely by new mutations. For a population in mutation-selection balance, however, this standing variation is correlated with the mutation rate (and even proportional to $U$ in the so-called house-of-cards regime; cf. Bürger 2000).

\section{Mode of reproduction}

There are indications that selection for robustness may be stronger in sexual and recombining populations than in clonal populations. Intuitively, this may stem from the additional genetic variation produced by sex and recombination that is experienced by a buffering gene (Stearns 1994). More formally, canalizing alleles have a direct fitness advantage in recombining populations, where genes can evolve relatively independently of the genetic background in which they accidentally originate. This has been demonstrated for robustness modifiers (Wagner et al. 1997). Although robustness modifiers do not increase the maximal fitness in the population (but rather decrease it if there is a cost of robustness), they nevertheless can spread if they increase its mean fitness. This will be the case if the fitness increase of mutant genotypes is sufficiently large that the marginal fitness of the modifier allele, averaged over all genetic backgrounds in the population, is positive. In contrast to mutation rate modifiers, which only have a fitness effect in the offspring generation, the fitness 
advantage of robustness alleles appears already in the parent generation. Robustness modifiers can therefore be selected even without linkage disequilibria.

In clonally reproducing populations, on the other hand, genes are linked to their genetic background. Since genetic robustness only increases the fitness of mutants, and not the fitness of wild types (with maximal fitness), robust wild types do not increase in frequency relative to less robust ones, as long as there is no back mutation from mutants to the wild type. In large asexual populations, robust wild types only spread due to the higher mutational backflow they receive from their fitter mutant neighbors. If robustness comes at a cost, only populations with high mutation rates and substantial back mutation will evolve robustness (Gabriel and Bürger 2000; Wilke et al. 2001; Hermisson et al. 2002).

The influence of recombination on robustness over an intermediate range of recombination rates is still controversial and difficult to assess. Presently, no systematic study exists for the invasion and maintenance of robustness as a function of recombination rate.

\section{Strength of selection}

In contrast to the frequency of the mutational perturbations, their fitness effects have surprisingly little impact on the potential for robustness to evolve, at least in mutation-selection balance. Intuitively, one might assume that traits that are under stronger stabilizing selection should have a stronger tendency to be buffered. In mutation-selection balance, however, the influence of the strength of stabilizing selection on the trait itself is found to be weak or even absent, depending on the parameter values (Wagner et al. 1997). This results from two opposing effects of stabilizing selection: it increases the fitness effect of the mutations present in a population, but simultaneously reduces the frequency of mutants in the equilibrium population.

\section{Population size}

Since the selective advantage of robustness alleles is of the order of the mutation rate (see above), the population size must be $N>1 / U$ for selection to be effective. This would make the evolution of robustness, as driven by recurrent mutations, impossible in small populations. As shown in a recent study by Krakauer and Plotkin (2002), however, robustness may still evolve in small populations by other means. In their model, the genetic load is increased by drift, leading to an elevated selective advantage of alleles that confer robustness against the negative fitness consequences of drift. Krakauer and Plotkin argue that small populations will adopt a strategy of 'hiding mutations from selection' by evolving buffering mechanisms, in contrast to large populations, where optimality is easier preserved by selectively removing mutations from the population. The relative importance of these two effects of population size depends on the geometry of the fitness landscape, which sets the terms for the invasion of new robust mutants.

\section{Congruent Scenarios}

Since environmental perturbations are frequent and diverse, opportunities for the evolution of environmental robustness abound. The much stronger impact of environmental perturbations relative to mutational perturbations on the phenotype is 
shown by the mutational heritability $h_{\mathrm{m}}{ }^{2}=V_{\mathrm{m}} / V_{\mathrm{e}}$ (i.e. the ratio of the variance due to new mutational and environmental input per generation), which is generally estimated to be of order $10^{-3}-10^{-2}$ (Houle et al. 1996). If mechanisms buffering against environmental fluctuations are also effective against mutational perturbations, as recently argued in particular by Meiklejohn and Hartl (2002), then genetic robustness may evolve by congruence. A congruent view of the evolution of robustness is also supported by a computational study by Ancel and Fontana (2000), who found that RNA shapes that are robust against environmental (thermodynamic) perturbations are also robust against mutational perturbations. Further support comes from recent studies of heat-shock proteins, such as Hsp90 and GroEL. These proteins are thought to have evolved to protect organisms from environmental and developmental perturbations, but appear to also buffer against genetic perturbation in Drosophila (Rutherford and Lindquist 1998), Arabidopsis (Queitsch et al. 2002), and Escherichia coli (Fares et al. 2002).

\section{Intrinsic Scenarios}

Phenotypic buffering might also emerge from global gene network properties like its connectivity or developmental stability. As first observed by A. Wagner (1996), and recently by Siegal and Bergmann (2002), developmental stability and mutational robustness are correlated in certain network models. Siegal and Bergmann (2002) argue that selection for increased developmental stability may be the main cause for the evolution of mutational robustness. Note that developmental stability, as defined by these authors, refers to the convergence of a deterministic dynamical system as a model of development. This dynamical system depends on the genotype, but not on the environment or developmental noise. The term 'developmental stability', therefore, should be clearly distinguished from developmental robustness, which refers to the stability under developmental noise. Evolution of genetic robustness in these systems is therefore an intrinsic property of the network model, rather than a consequence of buffering against non-heritable perturbations (i.e. congruence). Whether genetic robustness is a generic property of developmentally stable gene networks, is an open question. More work on a wider range of network architectures is required.

In general, network robustness can be adaptive, congruent or intrinsic in the above sense. Suppose that a gene network consists of any number of relatively independently evolving parameters (e.g. regulating enzyme concentrations, diffusion constants, etc.). In this parameter space, values for which the network is functional map to high-fitness plateaus and values that produce dysfunctional networks lie outside such plateaus. The robustness of a population with respect to mutations depends on the position of the population on the high fitness plateau. A population that is mostly concentrated in the interior of 'wide parts' of the plateau will be much less sensitive than a population that is distributed over the narrower parts where mutations easily push individuals over the edge. The overall shape of the plateau then speaks to the question of adaptive or intrinsic robustness. If most of the surface area of the plateau consists of narrow parts, then the function is not intrinsically robust. Over the course of evolution, the population will most likely first enter the plateau at one of the narrow parts with low functional robustness. Adaptive robustness ensues if selection subsequently moves the population towards the wider parts. Alternatively, if 
the entire plateau is wide, then function and robustness are coupled. Robustness thus arises as an intrinsic byproduct of the adaptive evolution of the network. If we add environmental perturbation to this picture, then we change the shape of the highfitness plateau. If these changes effectively narrow the plateaus, then genotypes that maintain functionality (i.e. remain on the plateau) under such perturbations will be those that lie in the 'inner parts' of the network. Thus genetic robustness may evolve via congruence (Ancel and Fontana 2000).

These alternatives have been studied in the segment polarity and the neurogenic networks of Drosophila (von Dassow et al. 2000; Meir et al. 2002). Simulations show that robustness emerges as an unselected and unexpected byproduct in the simplest models of a functional network. Recent studies (von Dassow, unpublished), however, also reveal the possibility that less robust rudimentary networks could have been evolutionary predecessors of final, more robust ones. The use of naturally occurring gene networks is a promising direction for the future study of mechanisms and processes that underlie network robustness.

A more quantitative use of phenotype landscapes to study robustness closely related to the above picture has been suggested by Rice (1998) and Nijhout (2002). If the trait plotted is defined as a smooth function of any number of environmental and genetic parameters, the slope of the landscape along a particular parameter axis is a measure of the sensitivity of the phenotype for variations in that parameter. Evolution towards regions with shallow slopes then means evolution of robustness. Note, however, that we do not agree with Nijhout's suggestion to use the correlation between variation in the trait and the parameter as a measure of robustness. This correlation will be 1 whenever all of the points in a plot of the trait against the parameter axis lie along a straight line, regardless of the slope of that line.

\section{WHICH TRAITS SHOULD EVOLVE GENETIC ROBUSTNESS?}

Robustness can evolve at different levels of complexity. At the lowest level, the product of a single gene may be buffered against the effects of mutations, for example, by dominance. Traits at other levels of organismal organization may also be robust, including products of a single developmental or metabolic pathway, morphological traits, larger functional units or modules, or even the overall fitness of the organism. At which of these levels of organization would we expect buffering to be most prominent?

\section{Size of the Genomic Basis}

Mechanisms at a low organizational level (e.g. single genes or pathways) can only buffer against relatively small numbers of mutations. Even if selection on a small unit is strong and the mutations affect multiple functions through pleiotropy (single genes affecting multiple traits), there may not be strong selection for robustness (see Adaptive Scenarios). The small number of mutations buffered against by dominance, and the small selection coefficients that result (of the order of the locus mutation rate), have been the primary arguments against Fisher's adaptive hypothesis. This may not, however, be a problem for mechanisms buffering larger organizational units (e.g. involved in global traits like body size). Such units have larger genomic 
bases, and hence suffer higher per-unit mutation rates. The same holds true for mechanisms that buffer many units at once. For example, heat-shock proteins chaperone a variety of enzymes (Rutherford 2003), and thus buffer against mutations in all the genes underlying these enzymes. If genetic variation is entirely produced by mutation-selection balance, then the selection coefficient for robustness can be as large as the overall deleterious mutation rate of the buffered unit or units.

\section{Level of Integration}

In principle, selection coefficients for buffering mechanisms of some lifehistory traits, which have very large genomic bases, could be quite high, close to the deleterious mutation rate for the entire genome. A trait like viability, however, may depend on several independent genetic components, each with a unique set of participating genes. Hence a single adaptation that buffers all potential sources of genetic variation is very unlikely. The effectiveness of a buffering mechanism thus depends on the level of genetic integration underlying the buffered unit. If, for example, several gene products feed into a common developmental or metabolic pathway, mutations in these genes might be buffered against by a single feedback loop.

\section{Directional or Stabilizing Selection}

If robustness is adaptive or congruent, its evolution may be hampered by directional selection on the targeted trait. This is particularly a problem when the buffering mechanism reduces the effects of deleterious and beneficial mutations alike. If so, and if the ratio of beneficial to deleterious mutations is relatively high, then the trait may evolve anti-robustness instead (Layzer 1980; see also Kawecki 2000). Even when beneficial mutations are not affected, the evolution of adaptive robustness will likely be secondary to trait adaptation itself. As envisaged by Rendel (1967), buffering mechanisms may evolve for a specific trait value and be ineffective if the trait mean changes. As long as the optimum value changes, a population will then simply evolve uphill in the fitness landscape, regardless of any changes in robustness along the way. Only after the population reaches a (local) fitness maximum, it may evolve along the fitness ridge, accumulating robustness (cf. Rice 2000). Hence, traits experiencing long episodes of stabilizing selection are natural candidates for adaptive robustness, whereas traits under directional selection will evolve adaptive robustness only after a selection plateau has been reached. Intrinsic robustness, on the other hand, is a byproduct of trait adaptation, and thus requires directional selection to evolve.

\section{Constraints}

For larger units, such as polygenic traits, constraints and conflicts between components may impede the buffering of the unit as a whole. Theoretical studies show that selection to keep a trait near its optimum may effectively impede the simultaneous buffering of all loci that contribute to the trait (Hermisson et al. 2003). As a consequence, the trait as a whole will not evolve complete robustness. Rather, 
only subunits (single genes, or sets of well-integrated genes that contribute to the trait but do not comprise its entire genetic basis) may become robust. The subunits that are most likely to evolve robustness are the ones with high mutation rates, often at the expense of reduced robustness of genes or subunits with lower mutation rates. This leads to a negative correlation between the subunit mutation rate and the effects of mutations occurring within the subunit. At the level of the entire trait, robustness often even decreases due to the large mutational effects that result as a side effect in subunits with small mutation rates.

\section{Robustness of Fitness}

Should we expect fitness, as a trait, to evolve genetic robustness? In general, any trait will evolve robustness when doing so also increases fitness. In an intrinsic scenario, this occurs when the robustness of a trait is intrinsically connected to improving its function. In the adaptive scenario, robustness of a trait evolves when this significantly reduces the effect of deleterious variation - and therefore also the robustness of fitness may be increased. This seems to make robustness of fitness the primary target of adaptive genetic robustness, and a particularly interesting trait to study in this context. Nevertheless, the answer to the above question is more complex.

There are indeed several factors that may favor the adaptive robustness of fitness. First, because of the large number of genes contributing to fitness, the rate of mutations with deleterious effects on fitness is higher than that for any other phenotypic trait. Second, if fitness is a continuous function of lower-level trait values, then robustness evolved for all lower-level traits will, almost necessarily, translate into robustness at the level of fitness. Third, even anti-robustness at the level of fitness components (e.g. increased fitness differences between zygotes due to selective abortion of zygotes with high mutation loads in plants and animals) may enhance robustness at the level of fitness. At first, it also seems that robustness of fitness should readily evolve, simply because mutations affecting fitness experience stronger selection than other mutations. For populations evolving at an equilibrium between mutation and selection, however, this is not true as the number of deleterious mutations is inversely related to their average fitness effect (see Strength of selection).

There are also several factors that hinder the evolution of robustness in fitness. First, since fitness is the only trait under long-term directional selection, a relatively large fraction of mutations has beneficial effects. Only in rare cases where populations adapt to a constant environment over longer periods - as perhaps in the long-term evolution experiment with $E$. coli of R.E. Lenski and coworkers (Lenski and Travisano 1994), might modifiers that increase robustness of fitness spread by natural selection. Second, since fitness is the ultimate product of so many genes, pleiotropy leading to trade-offs in the robustness of constituent subunits will be abundant. These trade-offs will severely constrain the potential for fitness robustness (cf. Constraints) as non-robust traits may contribute to an overall non-robustness in fitness. Non-robustness of constituent traits may result from directional selection or from a small genetic basis that allows genetic drift to outweigh selection for robustness. Such traits may still be under strong selection, and thus contribute significantly to reduce the net robustness of fitness. As a result, even if robustness of fitness increases on average this may be at the expense of an increased variation of 
robustness, which makes the detection of adaptive robustness at the level of fitness a problem for experimentalists.

Finally, we discuss a limitation that hinders many studies of the evolution of robustness. Most population genetic models of robustness use a fitness function that reflects stabilizing selection on an underlying phenotypic trait. In this case, robustness of fitness arises through the evolution of robustness at the trait level. Studying the evolution of robustness through these models is critical to understanding the changes in genotype and phenotype during evolution. It is clear, however, that robustness of fitness is not entirely explained by robustness at the level of an underlying phenotypic trait. Organisms can evolve organizational schemes, for example, that confer robustness through the modulation of different underlying phenotypic traits. In this case, fitness invariance can arise not through the invariance of underlying traits, but through changes in the importance of underlying phenotypic traits. Consider an organism that feeds on a primary nutrient. Robustness may arise through the evolution of reliable performance in the metabolic pathway that utilizes the nutrient. Alternatively, robustness may arise through the evolution of regulatory and structural machinery that allows the organism to use different metabolic pathways when the primary pathway is not performing well. Both scenarios involve robustness at the fitness level but may require separate theoretical treatments. In this paper, we focus on the first case but recognize that the evolution of new functions is critical to a comprehensive theory of genetic robustness and an important challenge for the future.

\section{PROXIMATE CAUSES OF ROBUSTNESS}

Genetic robustness can be achieved through (i) buffering against genetic perturbation or (ii) avoiding this perturbation. Perturbations are avoided, for example, by reducing the number of genes coding for a function, and eliminating somatic mutations in multi-cellular organisms. Some of these latter strategies are essentially anti-robust at a lower level (e.g. cell viability), but lead to robustness at a higher phenotypic level (cf. Krakauer and Plotkin 2002). (We consider perturbation avoidance also as a means to robustness, as it is an evolved response to the perturbations, which would persist without this response.) Buffering mechanisms are by definition epistatic, i.e. the buffering effect is the result of an interaction between the mutation (whose effect is buffered against) and the genetic background. We will use the term 'buffering epistasis' to mean that the phenotypic effect of multiple mutations is larger than the sum of the effects of each single mutation. If mutational effects are unidirectional, like mutations negatively affecting the fitness of a welladapted wild-type, buffering epistasis is equivalent to synergistic epistasis.

\section{Redundancy}

Buffering epistasis can result from redundancy, that is, interactions among genes with similar function (e.g. due to a recent duplication event), or from interactions among functionally unrelated genes (e.g. Wilkins 1997, 2002). Which of these causes is more important? Mutations that completely eliminate the function of an individual gene have very weak phenotypic effects in standard laboratory assays. It is tempting to attribute this observation to gene redundancy, since genomes contain 
many duplicated genes. However, the fact that in Saccharomyces cerevisiae at least $40 \%$ of the genes where knock-out mutations have exceedingly small fitness effects are single-copy genes (A. Wagner 2000; Gu et al. 2003), suggests that gene redundancy is not the only cause of genetic robustness. However, gene redundancy certainly contributes to robustness. For example, Gu et al. (2003) show that a knockout mutation of one gene in a duplicate pair is $20 \%$ more likely to have a weak fitness effect than a knock-out mutation of a single-copy gene. It bears mentioning that the gene-knockout approach is limited by the artificial environment of the laboratory, that is, knock-out mutations with small fitness effects in one particular laboratory environment, may have formidable fitness effects in the wild. Alternatively we can turn to molecular evolution. More specifically, if gene duplications play a major role in genetic robustness, then genes from large gene families should experience relaxed selective constraints, which would results in a high ratio of nonsynonymous to synonymous substitutions, $K_{\mathrm{a}} / K_{\mathrm{s}}$ ( $\left.\mathrm{Li} 1997\right)$. The evidence is mixed: in some fully sequenced eukaryotes a statistical association between gene family size and $K_{\mathrm{a}} / K_{\mathrm{s}}$ exists, but in others it is absent (Conant and Wagner 2002). In sum, while gene redundancy may play some role in genetic robustness, epistatic interactions among unrelated genes may be an equally important buffering mechanism.

Except from redundant genes, robustness can also result from redundant proteins. For example, Eldar et al. (2002) found that development involving the bone morphogenic pathway (BMP) in Drosophila is robust against variation in gene dosage as a result of the storage of excess signaling molecules. Slow diffusion from this buffer of signaling molecules appeared to result in a stable gradient of the morphogen.

\section{Epistasis Between Unrelated Genes}

Buffering epistasis is a general term that refers to many different patterns of genic interactions. For example, the genes with a direct effect on the trait (in the wildtype) and the genes involved in buffering these effects may be separate. The simplest and perhaps best-known example is the interaction between chaperones, such as heatshock proteins, and the enzymes whose function they buffer. Chaperones are thought to 'help' other enzymes fold into their functional structures, which is particularly beneficial after a heat shock (Rutherford 2003). The latter was evidenced by a faster recovery of fitness after a heat shock of a Drosophila line with multiple copies of Hsp70 (Feder et al. 1996). Disruption in heat-shock protein Hsp90 has revealed hidden genetic variation in Drosophila (Rutherford and Lindquist 1998) and Arabidopsis (Queitsch et al. 2002), while overexpression of heat-shock protein GroEL resulted in the recovery of fitness of $E$. coli strains that had accumulated deleterious mutations (Fares et al. 2002). These results suggest that chaperones not only buffer against environmental changes, but also against genetic variation at other loci. It is tempting to view these enzymes as secondary adaptations, favored by natural selection entirely for their ability to buffer other functions. Since heat shock proteins often also have other vital functions in the cell even under unperturbed conditions (cf. Feder and Hofmann 1999), however, this conclusion is not as obvious as it may seem. Detection of a buffering mechanism that has no other function, or may even be costly, would be excellent evidence for adaptive or congruent genetic robustness. 


\section{Epistasis Between Genes Encoding the Trait}

Buffering epistasis can also occur through genes that are simultaneously involved in both producing and buffering the trait. This architecture is very likely if robustness is intrinsic and evolves as a side effect of the trait function itself, but may also pertain to adaptive robustness. Indeed, Hartman et al. (2001) conclude from an analysis of double mutations in the yeast $S$. cerevisiae that buffering most often occurs by a small number of genes that function in the biological process that produces the trait being buffered. Theoretical approaches to studying robustness arising from epistatic interactions among the primary genes include phenotype landscape models (Rice 1998, 2000) and network models. In metabolic or developmental networks, complex epistatic interactions with buffering properties, as well as feed-back and feed-forward loops (Edwards and Palsson 2000; Shen-Orr et al. 2002) are common motifs. These loops enable a relatively constant metabolic flux despite fluctuations in substrate concentrations or input signals. As Wilkins (2002) points out, there are several properties (degeneracy and redundancy) that convey genetic robustness to promoter regions that regulate gene expression and, as such, function as the nodal points of developmental pathways.

More complex patterns of epistasis, involving networks of several to many interacting genes, may also lead to robust developmental output or phenotypes. A relatively simple example is the segment polarity network of insects, leading to developmentally robust modules, i.e. developmental subroutines leading to reiterated structures such as abdominal segments (von Dassow et al. 2000; Meir et al. 2002).

\section{ROBUSTNESS AND EVOLVABILITY}

By definition, genetic robustness reduces the degree to which the phenotype is influenced by underlying genetic variation. Since adaptive evolution requires phenotypic variation, a reduction of evolvability (future rate of evolution) may be a natural consequence of genetic robustness. Waddington originally proposed canalization as an explanation for the invariance of the adult phenotype. In this view, genetic robustness acts as a variational constraint that itself may be the result of natural selection (Maynard Smith et al. 1985). After a shift of the trait optimum (e.g. due to a change in the environmental conditions), this constraint must be overcome before the population can evolve toward the new optimum. Indeed, as can be seen from the work of Ancel and Fontana (2000), robustness may preclude mutation toward improved phenotypes and lead to an evolutionary dead end in which the phenotype (a computationally determined RNA secondary structure) is locked into a sub-optimal state. In fact, the relationship of robustness and evolvability is unclear. There are a number of realistic scenarios in which mutational buffering leads to increased evolvability.

\section{Versatility}

The first scenario pertains to traits other than the robust trait itself. Even if further evolution of the robust trait is slowed or halted, its robustness may facilitate adaptations of pleiotropically related characters if the robustness buffers against the 
deleterious side-effects of such adaptations (Baatz and Wagner 1997). Robustness may also promote the integration of the trait into higher organizational units. This idea rests on the enhanced versatility of robust units (i.e. 'modules'), as they are functional in a variety of genetic backgrounds. Such an increase in the evolvability of the genetic background of a robust trait may even confer a selective advantage on the trait itself by reducing the interdependence of components (Kirschner and Gerhart 1998). In this case, robustness evolves due to the adaptive advantage of increased evolvability. The putative evolutionary forces that drive this process, however, tend to rely on group level selection and are therefore generally weak (see Rutherford 2003, for a different view). Nevertheless, genic selection for increased evolvability is possible (G. P. Wagner, unpublished).

\section{Neutrality}

Extensive mutational robustness is tantamount to a mutationally connected network of genotypes exhibiting the same phenotype, a so-called 'neutral network' (Schuster et al. 1994). When an improved phenotype is not accessible through mutation from the current genotypes comprising a population, drift can move the population on the neutral network to far away regions in genotype space, thereby preserving the existing phenotype but vastly increasing the probability of mutation to the advantageous phenotype. It may seem paradoxical that mutational robustness may enable rather than prevent evolutionary innovation. The neutral network model, however, resolves this paradox. Mutational robustness allows for the accumulation of phenotypically silent mutations that alter the genetic basis of the trait and may thus set the stage for phenotypically important mutations (Fontana and Schuster 1998). According to this view, robustness increases the long-term evolvability not only of pleiotropically related traits (cf. Versatility), but of the robust trait itself. A classical rugged fitness landscape lacks large constant-fitness plateaus (neutral networks), and is thus anti-robust. In such a landscape, a population can become confined to a region of genotype space in which it must wait almost forever for the simultaneous occurrence of the right combination of advantageous mutations.

When robustness is (also) environmental, robust genotypes may also possess a higher initial tolerance towards new environments. This facilitates a large number of possible adaptations, since exposure to multiple environments offers more dimensions for adaptation than a constant environment.

\section{Capacity}

The third perspective considers the hidden (unexpressed) genetic variation that can accumulate in the robust state (Rendel 1967; Gibson et al. 1999; Schlichting and Smith 2002). Robustness mechanisms are thought by some to be 'capacitors' of phenotypic variation (Rutherford and Lindquist 1998; Queitsch et al. 2002; Rutherford 2003). When such a mechanism breaks down, for example after an environmental change, hidden variation is released. Such variation may be the fuel for further evolution. If environmental perturbations are merely transient, however, increased evolvability will only be ensured if the production of variation becomes independent 
of environmental inputs (see also Hansen et al. 2000, for a discussion of the contribution of 'hide-and-release mechanisms' of genetic variation to evolvability).

Waddington observed the release of hidden variation followed by adaptive evolution in Drosophila crossvein formation, and called this seemingly Lamarckian phenomenon 'genetic assimilation' (1953). When pupae from a laboratory stock of wild-type D. melanogaster were heat shocked, a gap in the posterior crossvein of the wings (a phenotype not normally found in untreated flies) appeared in about $40 \%$ of the adults. Waddington successfully selected for crossveinlessness under heat-shock conditions and eventually produced adults with broken cross-veins even when not exposed to heat. Waddington explained this behavior in neo-Darwinian terms as the canalization of the wild phenotype. His crucial assumption is that the robustness mechanism buffers against genetic perturbations only up to a certain threshold disturbance, and breaks down under stronger disturbances. Under strong environmental perturbations combined with selection for a trait revealed by the breakdown in buffering, mutations of small effect that contribute to the reliable expression of the selected trait will be favored, and eventually lead to the production of the trait even in the absence of the perturbation.

Rendel was the first to address the potential consequences of this process on evolutionary rates (1967). Anticipating the view of Eldredge and Gould (1972), he argued that a 'punctuated' evolutionary trajectory would result from alternating periods of stasis and adaptation following the breakdown of buffering mechanisms and the release of adaptive variation under rare environmental conditions. Whether this process produces only a temporary evolutionary spurt or a long-lasting increase in evolvability, however, is an open question.

To date, the relative importance of these scenarios is unresolved. Neutral networks and their consequences have been well characterized in computational models of evolving RNA populations (for a review see Fontana 2002). Recent experiments by Schultes and Bartel (2000) have also clearly demonstrated the existence of neutral paths in RNA sequence space. Yet the impact of mutational robustness on evolvability and evolutionary dynamics in general remains an important open issue that has yet to be explored experimentally.

\section{EXPERIMENTAL DETECTION OF ROBUSTNESS}

As with many problems in evolutionary biology, the experimental detection of robustness has taken two different forms. The comparative approach uses existing diversity to infer the existence and evolution of robustness. This approach benefits from strong signals produced by the long-term evolution of robustness, but suffers from a lack of a natural reference (i.e. the pre-robust ancestral state). The alternative approach, dubbed experimental evolution, studies the evolution of robustness by direct observation of short-term evolutionary processes (mostly using microbes) in the laboratory. This discipline benefits from the ability to perform control experiments and knowledge of the ancestral state, but suffers from insufficient time to evolve strong indications of robustness. Here we provide examples of both approaches. 
A useful measure of environmental robustness is the inverse of the environmental variance of a trait. In a heroic experiment, Whitlock and Fowler (1999) used 80,000 D. melanogaster flies to measure the phenotypic variance components of six wing size and shape characters after inbreeding. They observed an increase in the residual variance after inbreeding, as well as variation among lines in the level of residual variance. Since residual variance results primarily from environmental factors, these results suggest a genetic basis for developmental robustness (i.e. a particular form of environmental robustness that is directed towards fluctuations in the micro-environmental conditions that exist within an organism).

Another measure of developmental robustness is the fluctuating asymmetry (FA) of a trait. The amount of FA for any given trait can vary across both organisms and conditions (e.g. Clarke and McKenzie 1987), suggesting that evolution of this type of robustness is possible. However, low heritability estimates for FA and the finding of a mainly epistatic genetic basis of FA in many characters (see Leamy et al. 2002 , and references cited therein) shows that FA can not easily respond to selection in wild populations.

\section{Comparative Approach: Genetic Robustness}

Detecting genetic robustness has proven to be particularly difficult. The direct experimental determination of mutational variance by mutation-accumulation experiments of bottlenecked lines is extremely labor intensive. Although there have been a number of measurements (cf. Lynch et al. 1999), they have all been for wild type populations. Most estimates of genetic robustness rely on some form of indirect evidence.

\section{Genetic instead of mutational variance}

Instead of measuring mutational variance, several robustness studies have estimated genetic variance. Following Waddington (1953), genetic robustness has been primarily inferred from the increase of the genetic variance after a major mutation or exposure to an environmental challenge during development (such as heat shock). The classical measurements include wing- and cross-vein interruptions and scutellar bristle numbers in D. melanogaster, ocelli in D. subobscura, and vibrissae number in mice, all discussed in detail by Scharloo (1991). In all of these experiments, a character with almost vanishing phenotypic variance in the wild showed significant variation after a major mutational or developmental perturbation. The released variation responded positively to artificial selection, suggesting that the variation had a genetic basis. Inbred lines, however, did not respond to artificial selection, suggesting that the increased variance stemmed from unexpressed (hidden) variation already present in the base population. Such genetic variation with no phenotypic consequences under natural conditions might be construed as evidence for genetic robustness.

There is, however, a concern with this interpretation. None of the experiments described can discriminate between more or just other loci that are expressed as a result of the perturbation. In both cases, the (phenotypically expressed) genetic variation sharply increases due to the release of variation at loci that are neutral in the wild type. When other (but not more) loci are expressed after the perturbation, there 
will also be a reduction in the genetic variance due to loci that are expressed in the wild, but not in the perturbed state. However, since variation at these loci in the wild type is reduced by selection, this reduction may be negligible, resulting in a net increase in the genetic variance in both cases. If the same number of loci is expressed under both conditions (the 'other' scenario), the mutational variance will not necessarily be larger for the perturbed relative to the unperturbed state, and may even be smaller. Hence, the wild type is not robust. The original experiments indeed provide evidence for alleles that are expressed only in the wild type (Hermisson and Wagner, in preparation).

The release of selectable hidden variation also supports the claim that heatshock proteins Hsp90 (Rutherford and Lindquist 1998; Queitsch et al. 2002) and GroEL (Fares et al. 2002) act as genetic buffers. For this particular molecular mechanism, variation in the mutant (with knocked-out heat-shock function) is probably due to the activity of more alleles rather than simply a shift to other alleles.

A variation of this approach avoids some of the bias caused by using genetic instead of mutational variance. Mutations are introduced into inbred lines from multiple populations, and robustness is then estimated by the change of the genetic variance among lines. If the differences among the wild lines are due to drift or selection towards different optima and are not diminished by selection toward a global optimum, this method does not suffer the problem explained above. Using this approach, Gibson and van Helden (1997) did not observe an increase in phenotypic variance of Drosophila haltere characters in $U b x$ mutants relative to wild-type lines, and hence found no support for genetic robustness. In contrast, a second study (Polacysk et al. 1998) found a large increase in among-line variability after two major mutations (EGFR and Sevenless) in Drosophila photoreceptor differentiation. It seems likely, however, that in this case of a conserved trait, there has been selection towards a global optimum, which has eliminated variance among the wild-type lines (leading to the same problems as discussed for the older experiments above).

\section{Lack of reference and control}

A general problem with the comparative approach to detecting environmental and genetic robustness is the lack of a reference state. When can we say that robustness has evolved? In principle, the appropriate reference is the ancestral state, i.e. the same genotype before evolution had the opportunity to generate robustness. Since the ancestral state is not directly available, however, the comparative approach relies on indirect evidence. Typically, the evolution of robustness in the wild-type is inferred from observations of higher variability in mutants. Alternatively, one can compare the wild-type mutational variances of different traits. Stearns et al. (1995) studied the genetic variation caused by transposable-element insertions for five traits with different impacts on fitness in D. melanogaster. They found that the resulting phenotypic variation was lower for traits with larger fitness effects, and took this as evidence for genetic robustness. However, Houle (1998) challenged this conclusion, claiming that the correlation between fitness impact and insertion variability may be confounded by variation in the mutational target size, i.e. the number of genes involved in these traits. In general, inferring robustness from a comparison of mutational effects in different traits instead of different genetic backgrounds is problematic, as long as the connection of these measurements to the variability of an ancestral reference state is unclear. 


\section{Synergistic epistasis}

Because of the causal connection between synergistic epistasis and genetic robustness (see PROXIMATE CAUSES OF ROBUSTNESS), evidence for the latter can, in principle, be won by measuring the former. There are several methods for detecting synergistic epistasis between deleterious alleles. The extreme form of synergistic epistasis, truncation selection, is the ability (of a trait) to buffer against mutational variation. An overall lack of synergistic epistasis among alleles affecting fitness has been shown in strains constructed to have varying numbers of genetic marker mutations (de Visser et al. 1997; Whitlock and Bourguet 2000) and transposon insertions (Elena and Lenski 1997). Similar methods could still be used, however, to identify patterns of epistasis in characters that have been under long-term stabilizing selection and are thus likely to have evolved genetic robustness.

\section{Experimental Evolution}

Recently, the relatively new discipline of experimental evolution has been applied to the study of robustness. The rapid generations, large populations and ease of experimental manipulation of microbes make possible the direct observation of long-term evolution in the laboratory. Using similar approaches, computational evolutionary biologists use individual-based computer simulations to study the evolution of robustness. By manipulating the evolutionary environment and genotype of the organisms, specific hypotheses can be tested on the basis of the evolutionary outcome. A crucial advantage of this approach over the comparative approaches, is that it provides a natural reference point for any measure of robustness, i.e. the ancestral state. Alternatively, control treatments (evolutionary conditions that are not expected to lead to the evolution of robustness) can provide an appropriate baseline.

Wilke et al. (2001; Wilke and Adami 2003) simulated the evolution of 'digital organisms' for 1,000 generations with a high or a low mutation rate. Ultimately the organisms with a high mutation rate occupied lower, but flatter (more robust) fitness peaks than the ones evolved with a low mutation rate. These data suggest that genetic robustness can evolve rapidly at high mutation rates and lend themselves to verification through evolution experiments in the laboratory.

Elena and Lenski (2001) undertook such an experiment using E. coli. They compared the fitness effects of 12 random insertion mutations in two genetic backgrounds, one adapted during 10,000 generations to a simple laboratory environment and the other, its unevolved ancestor. If genetic robustness evolved during those 10,000 generations, then the average fitness effect should be smaller in the evolved strain than in the ancestor. There was, however, no decrease in the deleterious effects of mutations in the evolved background. These results can be interpreted in two different ways. First, robustness did not evolve since the necessary conditions (e.g. the duration of the experiment and/or mutation rate) were not met. Alternatively, robustness did evolve, but only towards the kinds of mutations naturally encountered during evolution and not towards the insertion mutations applied afterwards. Moreover, fitness itself may not easily evolve genetic robustness (see Robustness of Fitness).

Fares et al. (2002) recently took an alternative approach using a classical mutation-accumulation experiment with $E$. coli. After 3,240 generations of mutation accumulation, fitness was almost halved, presumably due to the accumulation of 
deleterious mutations. When Fares et al. overexpressed the chaperone enzyme GroEL in these mutated lines, fitness recovered significantly, indicating that GroEL might buffer the deleterious effects of mutations. This overexpression, however, resulted in fitness recovery only in a nutrient-rich environment and not in minimal media, suggesting a cost to such mutational robustness.

In conclusion, experimental evolution with microbes and virtual organisms is a promising tool for the direct observation of the evolution of genetic robustness. Future approaches might include modifying the GroEL experiments to compare the evolution of GroEL expression in mutator and wildtype strains; and measuring the fitness advantage of robustness through competitions among microbial strains having and lacking various mechanisms (e.g. an enzyme structure that is robust against mutations) under both normal conditions and conditions that favor robustness (e.g. in a mutator background or under environmental perturbations).

\section{Alternative Ideas}

There are many other promising directions. For example, comparing the mutational variance to the environmental variance of homologous traits across species might shed light on the extent of congruence between environmental and genetic robustness. Similarly, by comparing mutational variance to mutation rate and, similarly, environmental variance to rate of environmental fluctuations, one can test the prediction that robustness is expected particularly in species experiencing frequent perturbations.

Artificially selected traits in old and modern crop hybrids may serve as a terrific test bed for genetic robustness hypotheses. Many modern crops have been deliberately selected for flatter norms of reaction with respect to growing conditions across years and locations. One can measure the extent to which these environmentally robust traits are also more robust against mutations, again testing the existence of congruence.

Finally, the in vitro selection of single enzymes may provide a revolutionary perspective on the evolution of robustness. Genes coding for novel enzymes with a clearly defined function (e.g. conferring resistance against an antibiotic) are produced using error-prone PCR. These new alleles are transformed into a bacterial host, where selection or screening of enzyme function is possible. The simple relationship between genotype (single gene encoding an enzyme) and phenotype (enzyme function), together with the ability to introduce mutations at much higher frequencies than would be bearable for living organisms, allows scientists to address various hypotheses about the evolution of robustness. For instance, one can measure the relationship between the level of enzyme activity and the robustness of enzyme activity towards various environmental (e.g., temperature, $\mathrm{pH}$ ) or genetic perturbations (random or directed mutations), thereby addressing the nature of the robustness. This is also an ideal system for studying the effects of mutation rate on the evolution genetic robustness.

\section{Conclusions}


We surveyed recent advances in the evolutionary study of genetic robustness, directed by the following three questions. First, what are the evolutionary causes of genetic robustness? We have proposed three evolutionary scenarios, i.e. robustness as driven by direct adaptive benefits, as intrinsic property of adaptations, or as congruent correlate of environmental robustness. While their general significance is unresolved, the specific requirements of each scenario are discussed. For an adaptive scenario, we found that high mutation rates, large populations and asexual reproduction generally favor the evolution of robustness. While the importance of intrinsic robustness is unknown, recent studies of metabolic and developmental networks promise new progress in this area. In many cases, a congruent scenario, where genetic robustness evolves as by-product of environmental perturbation, seems to be most likely, because (i) such perturbations are more frequent than genetic perturbations, and (ii) work on RNA folding and heat-shock proteins suggests that congruence mechanisms indeed exist. As to what phenotypes we expect to be most robust, the answer is more complex. Generally, traits under stabilizing selection that are encoded by many interacting genes seem the best candidates. However, there are several complicating factors, which we discuss for the phenotypic trait 'fitness'.

Second, what are the evolutionary consequences of genetic robustness? At first sight, one might expect robustness to slow down or even stop evolution of the trait involved, since it hides the fuel (genetic variation) needed for evolution. While this principle was proven correct in a theoretical study, both theory and data suggest that robust traits might in the long term show increased rather than decreased adaptive potential. The reasons for an increased 'evolvability' of robust traits include the accumulation of hidden genetic variation that may be useful for later adaptation, the buffering of pleiotropic side-effects of adaptations, and the increased potential for a neutral exploration of genotype space.

And finally, how can we detect and measure robustness? Two general approaches have been taken here. The classical approach ('comparative method') has been to look for robustness accumulated over the millions of years of biological evolution, for example inferred from the increase in genetic variance after a major mutation or environmental challenge. However, the evidence is often indirect and suffers from the lack of a non-robust reference. Recently, a more direct approach has been used, where populations of microbes are allowed to evolve in the laboratory over hundreds to thousands of generations. This approach, although its evolutionary potential is limited by time constraints, does not suffer from a lack of control and promises exciting new data and insights for a more comprehensive theory of the evolution of genetic robustness.

\section{ACKNOWLEDGMENTS}

The paper is a product of the workshop on Detection and Evolution of Genetic Robustness, held April 23-25, 2002, in the Santa Fe Institute (SFI), Santa Fe, NM. The workshop was organized by G. P. Wagner and J. Hermisson (Yale University) as part of SFI's foundation program on robustness and was sponsored by the David and Lucile Packard Foundation. Special thanks are due to Erica Jen (SFI) for making this workshop possible. 


\section{REFERENCES}

Ancel Meyers, L. W., and J. J. Bull. 2002. Variation as an adaptation to an uncertain world. Trends Ecol. Evol. 17:551-557.

Ancel, L. W., and W. Fontana. 2000. Plasticity, evolvability, and modularity in RNA. J. Exp. Zool. (Mol. Dev. Evol.) 288:242-283.

Baatz, M. and G. P. Wagner. 1997. Adaptive inertia caused by hidden pleiotropic effects. Theor. Pop. Biol. 51:49-66.

Bagheri-Chaichian, H. 2001. Evolution of mutational effects in metabolic physiology. PhD Thesis, Department of Ecology and Evolutionary Biology, Yale University, New Haven.

Bourguet, D. 1999. The evolution of dominance. Heredity 83: 1-4.

Bürger, R. 2000. The Mathematical Theory of Selection, Recombination, and Mutation. Wiley, Chincester.

Clarke, G. M., and J. A. McKenzie. 1987. Developmental stability of insecticide resistence in the blowfly: a result of canalizing natural selection, Nature 181:1081-1019.

Conant, G. C., and A. Wagner. 2002. GenomeHistory: a software tool and its application to fully sequenced genomes. Nucl. Acids Res. 30:1-10.

Cornish-Bowden, A. 1987. Dominance is not inevitable. J. Theor. Biol. 125: 333-338.

Debat, V. and P. David. 2001. Mapping phenotypes: canalization, plasticity and developmental stability. Trends Ecol. Evol. 16: 555-561.

de Visser, J. A. G. M., R. F. Hoekstra, and H. van den Ende. 1997. Test of interaction between genetic markers that affect fitness in Aspergillus niger. Evolution 51:1499-1505.

Edwards, J. S. and B. O. Palsson. 2000. The Escherichia coli MG1655 in silico metabolic genotype: Its definition, characteristics, and capabilities. Proc. Natl. Acad. Sci. USA 97:5526-5533.

Eldredge, N., and S. J. Gould. 1972. Punctuated equilibria: an alternative to phyletic gradualism. Pp. 82-115 in T. J. M. Schopf, ed. Models in paleobiology. Freeman, San Francisco.

Eldar, A., R. Dorfman, D. Weiss, H. Ashe, B.-Z. Shilo, and N. Barkai. 2002. Robustness of the BMP mophogen gradient in Drosophila embryonic patterning. Nature 419:304-308.

Elena, S. F., and R. E. Lenski. 1997. Test of interaction between deleterious mutations in E. coli. Nature 390:395-398.

----. 2001. Epistasis between new mutations and genetic background and a test of genetic canalization. Evolution 55:1746-1752.

Fares, M. A., M. X. Ruiz-González, A. Moya, S. F. Elena, and E. Barrio. 2002. GroEL buffers against deleterious mutations. Nature 417:398.

Feder, M. E., and G. E. Hofmann. 1999. Heat-shock proteins, molecular chaperones, and the stress response: Evolutionary and ecological physiology. Annu. Rev. Physiol. 61:243-282.

Feder, M. E., N. V. Cartaño, L. Milos, R. A. Krebs, and S. L. Lindquist. 1996. Effect of engineering $\mathrm{Hsp} 70$ copy number on $\mathrm{Hsp} 70$ expression and tolerance of ecologically relevant heat shock in larvae and pupae of Drosophila melanogaster. J. Exp. Biol. 199:1837-1844. 
Fisher, R. A. 1928. The possible modifications of the response of the wild type to recurrent mutations. Am. Nat. 62:115-126 and Two further notes on the evolution of dominance. Am. Nat. 62:571-574.

Fontana, W. and P. Schuster. 1998. Continuity in evolution: On the nature of transitions. Science 280:1451-1455.

Fontana, W. 2002. Modelling 'evo-devo' with RNA. BioEssays 24:1164-1177.

Gabriel, W. and R. Bürger. 2000. Fixation of clonal lineages under Muller's ratchet. Evolution 54: 1116-1125.

Gerhart, J. and M. Kirschner. 1997. Cells, Embryos and Evolution. Blackwell Science, Malden.

Gibson, G. and S. van Helden. 1997. Is Function of the Drosophila Homeotic Gene Ultrabithorax Canalized? Genetics 147:1155-1168.

Gibson, G., M. Wemple, and S. van Helden. 1999. Potential variance affecting homeotic Ultrabithorax and Antennapedia phenotypes in Drosophila melanogaster. Genetics 151:1081-1091.

Gibson, G. and G. Wagner. 2000. Canalization in evolutionary genetics: a stabilizing theory? BioEssays 22: 372-380.

Gu, Z., L. M. Steinmetz, X. Gu, C. Scharfe, R. W. Davis, and W.-H. Li. 2003. Role of duplicate genes in genetic robustness against null mutations. Nature 421: 6366.

Haldane, J. B. S. 1930. A note on Fisher's theory of dominance. Am. Nat. 64:87-90.

Hallgrímsson, B., K. Willmore, and B. K. Hall. 2002. Canalization, developmental stability, and morphological integration in primate limbs. Yearbook of Physical Anthropology 45:131-158.

Hansen, T. F., A. Carter, and C. Chiu. 2000. Gene conversion may aid adaptive peak shifts. J. theor. Biol. 207: 495-511.

Hartman IV, J. L., B. Garvik, and L. Hartwell. 2001. Principles for the buffering of genetic variation. Science 291:1001-1004.

Hermisson, J., O. Redner, H. Wagner, and E. Baake. 2002. Mutation-Selection Balance: Ancestry, Load, and Maximum Principle. Theor. Pop. Biol. 62: 9-46.

Hermisson, J., T. F. Hansen, and G. P. Wagner. 2003. Epistasis in polygenic traits and the evolution of the genetic architecture under stabilizing selection. Am. Nat. (in press).

Houle, D. 1998. How should we explain variation in the genetic variance of traits? Genetica 102/103:241-253.

Houle, D., B. Morikawa, and M. Lynch. 1996. Comparing mutational heritabilities. Genetics 143: 1467-1483.

Kacser, H., and J. A. Burns. 1981. The molecular basis of dominance. Genetics 97: 6639-6666.

Kawecki, T. 2000. The evolution of genetic canalization under fluctuating selection. Evolution 54: 1-12.

Keightley, P. D. 1996. A metabolic basis for dominance and recessivity. Genetics 143: 621-625.

Kirschner, M., and J. Gerhart. 1998. Evolvability. Proc. Natl. Acad. Sci. USA 95:8420-8427.

Krakauer, D. C., and J. B. Plotkin. 2002. Redundancy, antiredundancy, and the robustness of genomes. Proc. Natl. Acad. Sci. USA 99:1405-1409.

Layzer, D. 1980. Genetic variation and progressive evolution. Am. Nat. 115: 809-826. 
Leamy, L. J., E. J. Routman, and J. M. Cheverud. 2002. An epistatic genetic basis for fluctuating asymmetry of mandible size in mice. Evolution 56:642-653.

Lenski, R. E., and M. Travisano. 1994. Dynamics of adaptation and diversification: A 10,000-generation experiment with bacterial populations. Proc. Natl. Acad. Sci. USA 91: 6808-6814.

Li, W.-H. 1997. Molecular evolution. Sinauer Associates, Sunderland.

Lynch, M., J. Blanchard, D. Houle, T. Kibota, S. Schultz, L. Vassilieva, and J. Willis. 1999. Perspective: Spontaneous deleterious mutation. Evolution 53:645-663.

Maynard Smith, J., R. Burian, S. Kauffman, P. Alberch, J. Campbell, B. Goodwin, R. Lande, D. Raup, and L. Wolpert. 1985. Developmental constraints and evolution. Quat. Rev. Biol. 60:265-287.

Mayo, O., and R. Bürger. 1997. Evolution of dominance: A theory whose time has passed? Biol. Rev. 72:97-110.

Meiklejohn, C. D., and D. L. Hartl. 2002. A single mode of canalization. Trends Ecol. Evol. 17: 468-473.

Meir, E., G. von Dassow, E. Munro, and G. M. Odell. 2002. Robustness, flexibility, and the role of lateral inhibition in the neurogenic network. Curr. Biol. 12:778786.

Muller, H. J. 1932. Further studies on the nature and causes of gene mutations. Pp. 213-255 in D. F. Jones, ed. Proc. $6^{\text {th }}$ Intern. Congr. Genet. Banta, Menasha, WI.

Nijhout, H.F. 2002. The nature of robustness in development. Bioessays 24: 553-561.

Omholt, S., E. Plahte, L. Oyehaug, and K. Xiang. 2000. Gene regulatory networks generating the phenomena of additivity, dominance and epistasis. Genetics 155: 969-980.

Orr, H. A. 1991. A test of Fisher's theory of dominance. Proc. Natl. Acad. Sci. USA 88: 11413-11415.

Polaczysk, J. P., R. Gasperini, and G. Gibson. 1998. Naturally occurring variation affects Drosophila photoreceptor determination. Dev. Genes Evol. 207:462470.

Porteous, J. W. 1996. Dominance - one hundred and fifteen years after Mendel's paper. J. Theor. Biol. 182: 223-232.

Queitsch, C., T. A. Sangster, and S. Lindquist. 2002. Hsp90 as a capacitor of phenotypic variation. Nature 417:618-624.

Rendel, J. M. 1959. Canalization of the acute phenotype of Drosophila. Evolution 13:425-439.

----. 1967. Canalization and Gene Control. Logos Press, London.

Rice, S. H. 1998. The evolution of canalization and the breaking of von Bear's laws: modeling the evolution of development with epistasis. Evolution 52:647-656.

----. 2000. The evolution of developmental interactions: Epistasis, Canalization, and Integration. Pp. 82-98 in J. B. Wolf, E. D. Brodie III, and M. J. Wade, eds. Epistasis and the Evolutionary Process. Oxford University Press, Oxford.

Rutherford, S. L. 2000. From genotype to phenotype: buffering mechanisms and the storage of genetic information. BioEssays 22:1095-1105.

---. 2003. Between genotype and phenotype: protein chaperones and evolvability. Nat. Rev. Genet. 4:263-274.

Rutherford, S. L. and S. Lindquist. 1998. Hsp90 as a capacitor of morphological evolution. Nature 396:336-342. 
Savageau, M. A. 1992. Dominance according to metabolic control analysis: major achievement or house of cards? J. Theor. Biol. 154: 131-136.

Scharloo, W. 1988. Selection on morphological characters. Pp. 230-250 in G. de Jong, ed. Population genetics and Evolution. Springer, Berlin.

----. 1991. Canalization: Genetic and developmental aspects. Annu. Rev. Ecol. Syst. 22:65-93.

Shen-Orr, S. S., R. Milo, S. Mangan, and U. Alon. 2002. Network motifs in the transcriptional regulation network of Escherichia coli. Nature Genetics 31:6468.

Schlichting, C. D., and H. Smith. 2002. Phenotypic plasticity: linking molecular mechanisms with evolutionary outcomes. Evol. Ecol. 16:189-211.

Schmalhausen, I. I. 1949. Factors of evolution: The theory of stabilizing selection. Univ. Chicago Press, Chicago. (Reprinted in 1986.)

Schultes E. A. and D. P. Bartel. 2000. One sequence, two ribozymes: Implications for the emergence of new ribozyme folds. Science 289:448-452.

Schuster, P., W. Fontana, P. F. Stadler and I. Hofacker. 1994. From sequences to shapes and back: A case study in RNA secondary structures. Proc. Roy. Soc. (London) B 255:279-284.

Siegal, M. L., and A. Bergmann. 2002. Waddington's canalization revisited: developmental stability and evolution. Proc. Natl. Acad. Sci. USA 99:1052810532.

Stearns, S. C. 1994. The evolutionary links between fixed and variable traits. Acta Pal. Pol. 38:215-228.

Stearns, S. C., M. Kaiser, and T. J. Kawecki. 1995. The differential genetic and environmental canalization of fitness components in Drosophila melanogaster. J. Evol. Biol. 8:539-557.

Thatcher, J.W., J. M. Shaw, and W. J. Dickinson. 1998. Marginal fitness controbutions of nonessential genes in yeast. Proc. Natl. Acad. Sci. USA 95:253-257.

von Dassow, G., E. Meir, E. M. Munro, and G. M. Odell. 2000. The segment polarity network is a robust developmental module. Nature 406:188-192.

Waddington, C. H. 1953. The genetic assimilation of an acquired character. Evolution 7:118-126.

----. 1957. The strategy of the genes. MacMillan, New York.

Wade, M. J. 2002. A gene's eye view of epistasis, selection and speciation. J. Evol. Biol. 15: 337-346.

Wagner, A. 1996. Does evolutionary plasticity evolve? Evolution 50: 1008-1023.

----. 2000. Robustness against mutations in genetic networks of yeast. Nat. Genet. 24:355-361.

Wagner, G. P., G. Booth, and H. Bagheri-Chaichian. 1997. A population genetic theory of canalization. Evolution 51:329-347.

Whitlock, M. C., and K. Fowler. 1999. The change in genetic and environmental variance with inbreeding in Drosophila melanogaster. Genetics 152:345-353.

Whitlock, M. C., and D. Bourguet. 2000. Factors affecting the genetic load in Drosophila: synergistic epistasis and correlations among fitness components. Evolution 54:1654-1660.

Wilke, C. O., J. L. Wang, C. Ofria, R. E. Lenski, and C. Adami. 2001. Evolution of digital organisms at high mutation rates leads to survival of the flattest. Nature 412:331-333. 
Wilke, C. O., and C. Adami. 2003. Evolution of mutational robustness. Mut. Res. 522:3-11.

Wilkins, A. S. 1997.Canalization: a molecular genetic perspective. BioEssays 19: 257-262.

----. 2002. The evolution of developmental pathways. Sinauer, Sunderland.

Wright, S. 1929. Fisher's theory of dominance. Am. Nat. 63:274-279.

---- 1934. Physiological and evolutionary theories of dominance. Am. Nat. 68: 25-53.

----. 1977. The evolution of dominance. Pp. 498-526 in: Evolution and genetics of populations, Vol. 3. Univ. of Chicago Press. 